\title{
MAPPING THE GRAIN-BOUNDARY CHARACTER- (FREE) ENERGY LINKAGE IN POLYCRYSTALLINE MATERIALS
}

\author{
BRENT L. ADAMS \\ Department of Materials Science and Engineering, Carnegie Mellon University, \\ Pittsburgh, PA 15213-3890
}

(Received 10 January 1995)

\begin{abstract}
Relationships between structure-sensitive macroscopic properties and the grain boundary network are known to depend upon the intrinsic variability of the local physico-chemical properties of the boundaries and upon how these are distributed and connected in the network. Progress towards acquiring a data base linking the local properties of grain boundaries with their geometrical character (lattice misorientation and inclination) has been hampered by the rate at which grain boundary structure can be experimentally determined, by the opacity of polycrystalline materials, and by the very large number of physically distinctive (geometrical) characteristics of grain boundaries which occur in nature.

Described in this paper is a perspective on modern opportunities to solve the comprehensive inverse problem relating the thermodynamic properties of grain boundaries to their geometrical type over the full (five-parameter) fundamental zone. The advent of rapid, automated measurements of local lattice orientation (orientation imaging microscopy), when coupled with precision serial sectioning, makes it feasible now to characterize the local crystallographic and geometrical features of very large numbers of grain boundaries and triple junctions. Such data can be analyzed within a classical framework in order to extract the thermodynamic properties mapping. This paper examines the opportunities and challenges associated with the requisite measurements and analysis.

This paper is dedicated to the memory of Professor Hsun Hu, whose kind encouragement and penetrating insight has inspired and lifted a generation of young scientists.
\end{abstract}

KEY WORDS: Grain boundaries, free energy, grain boundary tension, chemical segregation, microdiffraction, orientation imaging microscopy

\section{INTRODUCTION}

The vast majority of technologically useful materials are of polycrystalline constitution. They consist of relatively perfect crystalline regions that are misoriented with respect to each other, and thus separated by a network of interfaces. The local physico-chemical characteristics of these interfaces are known to be strongly dependent upon the geometrical details of lattice misorientation and the orientation of the interface plane. However, the details of this dependence have been measured or simulated for only a relatively few boundaries in any alloy system.

We shall hereafter refer to the geometrical and crystallographic features of an isolated grain boundary as the "character" or "type" of the grain boundary. What is urgently needed is an association between the character and the local (intrinsic) properties of grain boundaries: not just for a few of highly symmetric character, but for the entire fundamental zone of geometrically distinctive types. These associations dictate many important macroscopic properties through the details of their distribution and 
connectivity in the microstructure (c.f. Lejcek and Hofmann, 1995). They also establish the subsequent evolution of microstructure through a complex interplay of the thermodynamic driving forces and kinetic constraints which determine the motions of grain boundaries. When the complete set of all possible grain boundary types and their associated local properties is of interest, we shall refer to the "mapping" between character and properties. The main focus of the paper is an exemplary mapping between grain boundary type and excess free energy.

The class of properties often referred to as "structure-sensitive" properties is known to be very sensitive to the details of the grain-boundary network structure. They are readily distinguished from elementary "structure-insensitive" properties by the fact that they are not related by simple volume-averages to the properties of the constituent crystalline phases. (Thus, they are more closely related to the misorientation distribution function (MDF) than to the orientation distribution function (ODF).) Also, they are generally non-linear, whereas many structure-insensitive properties are linear. Some of these extrinsic properties are associated with the most vexing challenges limiting the lifetime and reliability of engineered systems. Prominent examples include (at the large scale) localized stress-corrosion leading to cracking of steam generator components, and (at the microscopic scale) electromigration failure of interconnects in microelectronic circuits.

Notwithstanding mounting economic and social pressures to find solutions to these problems, progress towards understanding and controlling structure-sensitive properties has been slowed by a powerful impasse. The world community of investigators has thus far been able to explore only a small part of the mapping of character-properties relationships. The range of grain-boundary types naturally occurring in polycrystals comprises a very large space (called the fundamental zone, c.f. Sutton and Balluffi, 1995) defined by a minimum of five degrees of freedom (three fixing lattice misorientation and two fixing boundary inclination). Although this linkage has been explored for some individual boundaries, the total accumulation of these observations by the world materials community comprises only a tiny fraction of the natural space of interfacial structure. Inference based upon such limited data is commensurately limited in detail and reliability.

The structure of the grain boundary network is also of plenary significance to a better understanding of the evolution of polycrystalline microstructure during thermomechanical processing. Atomistic processes essential to the evolution of microstructure include grain boundary mobility, sliding and diffusivity. Efforts to develop realistic models for microstructural evolution have been impeded by the lack of information about these local boundary characteristics.

Thus, many future advances in materials technology must await a deeper, more extensive characterization of the structure of the interfacial network, including the mapping which links character to local properties, which is the sine qua non for future progress. This much broader level of inquiry could propel a revolution in the ability of the materials community to relate the microstructures of polycrystalline materials to their structure-sensitive properties and to engineer the processing of polycrystalline materials for optimal performance and stability in critical environments. The construction of such mappings is a natural extension of the traditional focus of modern texture analysis. 


\section{OPPORTUNITIES EMERGING FROM COMPUTER-AIDED ORIENTA- TION IMAGING MICROSCOPY}

A confluence of recent technological innovations in microscopy makes it possible now to characterize the mesoscale structure of polycrystals. Of particular importance are advances over the past three years in computer-automated scanning electron microscopy, especially orientation imaging microscopy (OIM) (Adams et al, 1993). These developments, when coupled with modern approaches to precision polishing for calibrated (serial) removal of flat sections from surfaces, now enable a fundamental leap in capability to characterize interfacial networks. Tomographic inquiry of the 3dimensional structure with spatial resolutions on the order of $100 \mathrm{~nm}$ are well within reach using modern techniques. Given that modern OIM is capable of interrogating as many as $10^{6}$ local lattice orientations in reasonable experimental times $(\sim 200 \mathrm{~h})$, it is evident that tomographic OIM is capable of interrogating the 3-dimensional mesostructure of very large numbers of grains, grain boundaries and triple junctions.

\section{REVIEW OF THE EQUILIBRIUM THERMODYNAMICS OF TRIPLE JUNCTIONS}

The basic thermodynamics of interest was introduced by Gibbs (1962) nearly a century ago. The important entity is the interface excess free energy (per unit area) $\sigma$, which (in the absence of interfacial strain) is a function of temperature $T$, pressure $P$, the chemical potentials of each component $\mu_{i}$, misorientation of the lattice between the adjacent grains $\Delta g$, and the unit normal describing inclination of the boundary $\hat{n}$. We seek to determine the functional dependence of $\sigma$ on the crystallographic parameters $\Delta g, \hat{n}$ for selected $T, P$ and $\mu_{i}$.

The main concept is illustrated by considering the geometry of triple junctions equilibrated under time and temperature combinations such that diffusion distances lie within the mesoscale range that is now accessible by tomographic OIM. Under such conditions the measurable geometry of the junction reflects a balance of the forces and torques acting on the adjacent boundaries. These are intimately related to the excess free energies associated with the boundaries. Following Herring (Herring, 1952; Mullins, 1963) each equilibrium triple junction is described by a system of two independent equations in six unknowns (three unknown interface energies, and three unknown torques):

$$
\sum_{j=1}^{3}\left\{\sigma_{j} \hat{b}_{j}+\left(\partial \sigma_{j} / \partial \chi\right) \hat{n}_{j}\right\}=0
$$

In this expression $\hat{n}_{j}$ is the unit boundary normal associated with the jth boundary in the triple junction. Similarly $\hat{b}_{j}$ is a unit vector lying perpendicular in the jth boundary and perpendicular to the triple line $\hat{l}=\hat{n}_{j} \otimes \hat{b}_{j}$ which is common to all three adjacent boundaries. The angle $\chi$ is defined to be the right-handed angle of rotation about $\hat{l}$.

The six unit vectors found in relations (1) obtain directly from experimental measurements of the orientation of the triple line and the three dihedral angles. Although it is not possible to extract interfacial energies from the equilibrium balance equations for an isolated triple junction, from large sets of triple junctions such an extraction is possible. Consider for example $\mathrm{N}$ triple junctions comprising $3 \mathrm{~N}$ interfaces. For simplicity, let all of these junctions have parallel directions. Suppose that the set of 
$3 \mathrm{~N}$ interfaces includes $\mathrm{M}$ distinctive types. Thus the number of unknown energies and torques in the set is $2 \mathrm{M}$. Herring's relations provide $\mathrm{K} \leq 2 \mathrm{~N}$ independent equations inter-relating these $2 \mathrm{M}$ unknowns. Clearly if $2 \mathrm{M} \leq \mathrm{K}$ a solution for all of the unknowns is possible (to within an unknown normalization factor). Thus, we expect to be able solve for the set of unknowns by searching the random polycrystal for a self-consistent set of triple junctions. In the broadest sense, if data from sufficient triple junctions are available, it will be possible to extract free energies for interfaces over the entire fundamental zone. The number of triple junctions which must be examined is dependent upon the desired angular resolution, and the conditioning and coverage of the input data.

From raw tomographic OIM data, a large system of Herring's relations (coupled firstorder linear differential equations) can be constructed. This system exhibits a tree structure owing to the fact that the equations for different junctions represent overlapping interface types. The complete system of equations may be divided into a collection of disjoint trees. Such a tree is a system of many equations with relatively few unknowns. With perfect information, a tree may be descended to iteratively solve for all the interfacial energies. However, there are many difficulties with this naive idea: the system will often be over determined, giving rise to inconsistent solutions; any error in the data due to noisy measurements could be propagated through the system since its solution may require successive division by many small numbers.

We are currently developing an innovative strategy in conjunction with the Center for Nonlinear Analysis at Carnegie Mellon University (D. Kinderlehrer) that we believe will be efficient and provide a reliable solution. It relies on two ideas: First, applying a least-squares technique, a new system will be derived which has the same number of unknowns as equations; this system is still very large and dense (most coefficients are not zero). The size of this system is a problem, however, since algorithms for solving large systems (with, say $10^{4}$ unknowns) are not practical for dense systems. The second idea is to study the large matrix of the tree and to use the expected values of the coefficients (and the joint distribution) to establish the coefficient matrices of subtrees of the main tree for the least-squares method. These smaller equations may be solved quickly, and need only be solved once. Simulations are being designed to test this strategy.

\section{APPLICATION OF THE GENERALIZED HARMONIC SERIES METHOD TO THE MAPPING PROBLEM}

For single-phase polycrystals the excess free energy can be expressed as a real-valued function on the fundamental zone of grain boundary structure, which is denoted here as $\Gamma / O(3) / \Gamma \otimes S^{2} / \Gamma$ where $\Gamma$ denotes the symmetry subgroup of the orthogonal group $O(3)$ associated with the crystal lattice of the material, and $S^{2}$ is the twodimensional manifold consisting of the surface of a unit sphere of directions in three dimensional real space $\Re^{3}$. $\Gamma / O(3) / \Gamma$ is the homogeneous double-coset space associated with all possible misorientations of a lattice with symmetry subgroup $\Gamma$, and $S^{2} / \Gamma$ represents the set of all possible physically distinctive boundary inclinations in the crystal lattice. Thus

$$
\sigma: \Gamma / O(3) / \Gamma \otimes S^{2} / \Gamma \rightarrow \Re
$$

where $\Re$ denotes the set of real numbers. 
Using the notation established by Bunge (1982), $\sigma$ can be expressed in terms of a series of harmonic basis functions $\ddot{\mathrm{T}}_{1}^{\mu v}(\Delta g) \dot{\mathrm{k}}_{\mathrm{r}}^{\eta}(\hat{\mathrm{n}})$ :

$$
\sigma(\Delta g, \hat{n})=\sum_{l=0}^{\infty} \sum_{\mu=1}^{M(l)} \sum_{v=1}^{M(l)} \sum_{r=0}^{\infty} \sum_{n=1}^{M(r)} S_{l \mu v}^{r \eta} \ddot{\mathrm{T}}_{1}^{\mu v}(\Delta g) \dot{\mathrm{k}}_{\mathrm{r}}^{\eta}(\hat{\mathrm{n}})
$$

Here $\ddot{T}_{1}^{\mu v}(\Delta g)$ denotes the generalized spherical harmonic functions, doubly symmetrized with respect to $\Gamma$, the symmetry subgroup of the lattice; and $\dot{k}_{r}^{\eta}(\hat{n})$ denotes the surface spherical harmonics, also symmetrized with respect to $\Gamma$. It is known (Courant and Hilbert, 1937) that the product of these constitutes a complete orthogonal basis on $\Gamma / O(3) / \Gamma \otimes S^{2} / \Gamma$.

Equation (3) can be directly introduced into Herring's relation (1) which can be organized in the following form:

$$
\sum_{j=1}^{3} \sum_{l=0}^{\infty} \sum_{\mu=1}^{M(l)} \sum_{v=1}^{M(l)} \sum_{r=0}^{\infty} \sum_{\eta=1}^{M(r)} S_{l \mu v}^{\eta} \ddot{\mathrm{T}}_{1}^{\mu v}\left(\Delta g_{j}\right)\left[\dot{\mathrm{k}}_{\mathrm{r}}^{\eta}\left(\hat{\mathrm{n}}_{\mathrm{j}}\right) \hat{b}_{j}+\left(\partial \dot{\mathrm{k}}_{\mathrm{r}}^{\eta}\left(\hat{\mathrm{n}}_{\mathrm{j}}\right) / \partial \chi\right) \hat{\mathrm{n}}_{\mathrm{j}}\right]=0
$$

Evidently, the coefficients $S_{l \mu \nu}^{r \eta}$ are taken to be functions of $T, P$ and $\mu_{i}$. Again it is important to emphasize that the derivatives in relations (4) are taken about the triple junction $\hat{l}$. The evaluation of the derivatives is a straightforward technical matter, which will not be discussed further here other than to point out that it has been partially addressed in the work of McLean and Gale (1969) and Gale et al. (1972), who have taken an approach which is similar to that outlined here to extract surface energies from equilibrium thermal (surface) grooves.

What is desired, of course is to extract the series coefficients $S_{l \mu \nu}^{r \eta}$ from a sufficiently large set of Herring's relations using (4). For each measured triple junction a set of three $\Delta g_{j}, \hat{n}_{j}$ and $\hat{l}$ are measured from which a system of two independent equations (4) can be constructed. If the series expansion for $\sigma$ is truncated in a manner such that there are $M$ unknown $S_{l \mu \nu}^{r \eta}$, then as before we require at least $2 \mathrm{~N} \geq \mathrm{M}$ Herring's relations, originating from $\mathrm{N}$ triple junctions, to solve for the desired coefficients.

\section{CONCLUDING REMARKS}

OIM, when coupled with precision serial polishing, is now capable of revealing the 3-dimensional structure of polycrystals to resolutions of about $100 \mathrm{~nm}$. When the temperatures and times of equilibration have been sufficient, the mesostructure revealed by this tomography can be expected to satisfy Herring's force and torque balance. Thus, the mesostructure and mesotexture carries data from which we can expect to extract a mapping relating grain boundary excess free energy to the character of the grain boundaries over the full fundamental zone, $\Gamma / O(3) / \Gamma \otimes S^{2} / \Gamma$. This is a classical inverse problem in materials science. Whereas from data on individual triple junctions it is not possible to extract the complete set of ratios of free energy for the adjacent boundaries, when many triple junctions are considered together a comprehensive extraction in possible. Although such an extraction will surely be very demanding in terms of the numerical and analytical approach, the benefits derived from complete character-free energy mappings could be substantial in relation to pressing issues of structure-sensitive properties and performance. 


\section{Acknowledgment}

The author wishes to acknowledge helpful discussions of this problem with W. Mullins and D. Kinderlehrer, both of Carnegie Mellon University.

\section{References}

Adams, B. L., Wright, S. I. and Kunze, K. (1993). Metallurgical Transactions, 24A, 819-831.

Bunge, H. J. (1982). Texture Analysis in Materials Science, Butterworths, London.

Courant, R. and Hilbert, D. (1937). Methods of Mathematical Physics, Volume 1, John Wiley and Sons, New York, 56.

Gale, B., McLean, M. and Hunt, R. A. (1972). Philosophical Magazine, 25, 947-960.

Gibbs, J. W. (1962). The Scientific Papers of J. Williard Gibbs, Volume 1, Thermodynamics, Dover Publications, New York.

Herring, C. (1952). in Structure and Properties of Solid Surfaces, R. Gomer and C. S. Smith, eds., University of Chicago Press, Chicago.

Lejcek, P. and Hofmann, S. (1995). Thermodynamics and Structural Aspects of Grain Boundary Segregation, CRC Critical Reviews in Solid State and Materials Science, 20, 1-134.

McLean, M. and Gale, B. (1969). Philosophical Magazine, 20, 1033-1045.

Mullins, W. W. (1963). in Metal Surfaces: Structure, Energetics and Kinetics, W. D. Robertson and N. A. Gjostein, eds., The American Society for Metals, Metals Park, OH, 17-66.

Sutton, A. P. and Balluffi, R. W. (1995). Interfaces in Crystalline Materials, Clarendon Press, Oxford. 